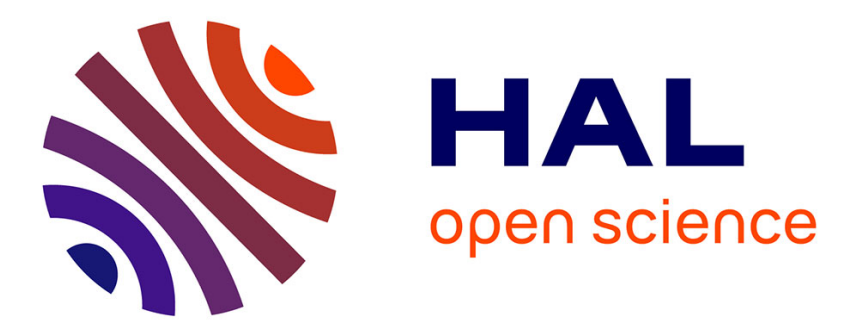

\title{
Multidisciplinary Interface Modelling: A Case Study on the Design of 3D Measurement System
}

Chen Zheng, Julien Le Duigou, Matthieu Bricogne, Peter Hehenberger, Benoît

Eynard

\section{- To cite this version:}

Chen Zheng, Julien Le Duigou, Matthieu Bricogne, Peter Hehenberger, Benoît Eynard. Multidisciplinary Interface Modelling: A Case Study on the Design of 3D Measurement System. 12th IFIP International Conference on Product Lifecycle Management (PLM), Oct 2015, Doha, Qatar. pp.856866, 10.1007/978-3-319-33111-9_78 . hal-01377516

\section{HAL Id: hal-01377516 https://hal.inria.fr/hal-01377516}

Submitted on 7 Oct 2016

HAL is a multi-disciplinary open access archive for the deposit and dissemination of scientific research documents, whether they are published or not. The documents may come from teaching and research institutions in France or abroad, or from public or private research centers.
L'archive ouverte pluridisciplinaire HAL, est destinée au dépôt et à la diffusion de documents scientifiques de niveau recherche, publiés ou non, émanant des établissements d'enseignement et de recherche français ou étrangers, des laboratoires publics ou privés. 


\title{
Multidisciplinary Interface Modelling: a Case Study on the Design of 3D Measurement System
}

\author{
Chen Zheng ${ }^{1}$, Julien Le Duigou ${ }^{1}$, Matthieu Bricogne ${ }^{1}$, Peter Hehenberger ${ }^{2}$, Benoît \\ Eynard $^{1}$, \\ ${ }^{1}$ Sorbonne Universités, Université de Technologie de Compiègne \\ Department of Mechanical Systems Engineering, UMR CNRS 7337 Roberval \\ CS 60319, 60203 Compiègne Cedex, France \\ \{chen.zheng; julien.le-duigou; matthieu.bricogne; benoit.eynard\}@utc.fr \\ ${ }^{2}$ Institute of Mechatronic Design and Production \\ Johannes Kepler University Linz, Linz, Austria \\ peter.hehenberger@jku.at
}

\begin{abstract}
Nowadays, a mechatronic system is regarded as a synergistic integration of a wide range of disciplines (electrical/electronic, mechanical, software). Therefore, an integration of numerous technical disciplines and expertises is often required during the design process of mechatronic systems. However, neither academia nor industry has yet provided fully effective solutions to help the engineers achieve such multidisciplinary integration. Multidisciplinary interface modelling approach is considered as an effective way to represent the interaction between the boundaries of components of different disciplines. The authors propose a new multidisciplinaryinterface modelling approach to address the issue of multidisciplinary integration. A three dimensional (3D) measurement system, considered as an optomechatronic system integrating synergistically the electrical/ electronic system, mechanical parts and information processing and optical technology is used to demonstrate the multidisciplinary interface modelling approach. A demonstrator based on the proposed approach has been developed by using CATIA System Engineering V6.
\end{abstract}

Keywords: Mechatronics Design, Multidisciplinary Integration, Interfaces.

\section{Introduction}

Mechatronic systems are considered as the resulting integration of electrical/electronic systems, mechanical parts and information processing [1]. Because mechatronic systems encompass a wide range of disciplines, therefore the multidisciplinary integration has been proposed and it becomes more and more crucial for mechatronic systems $[2,3]$.

To achieve such multidisciplinary integration, one of the problemswhich should be overcome is described as "Design data-related problems" [4]. Such "Design datarelated problems" are related to the edition and management of the diversity of product data from different disciplines. However, neither academia nor industry has 
yet provided relevant solutions to solve the problems in design of mechatronic systems[5].

The concept "interface" in the context of interface modelling of mechatronic systems refers to any logical or physical relationship required to integrate the boundaries between systems or between systems and their environment [6]. These multidisciplinary interfaces can be also used to indicate the collaboration of engineers and to provide a high-level guidance for organisation of design process. The authors propose a multidisciplinary interface modelling approach to address the issue of multidisciplinary integration. The proposed multidisciplinary interface modelling approach includes three parts: interface classification, interface data model and interface compatibility.

Section 2 will give a review of related work on current interface modelling approaches. Then, an overview on the multidisciplinary interface modelling approach will be provided in Section 3. A 3D measurement system will be introduced as the case study in Section 4. The design of pattern projection sub-system which is considered as one of the most important parts in the measurement systemwill beselected and detailed by using the proposed approach. Finally, the authors will draw the conclusions and point out the future prospects for the multidisciplinary interface modelling approach.

\section{Related work}

From the mid 1980s, the interface between systems and subsystems has been widely used in software engineering [7]. During software design process, separated modules of a program execute one aspect of the desired functionality. Such modules interact with each other through interfaces. As systems became increasingly complex, a complex system was always decomposed into subsystems. The topic of interface is at the heart of the multidisciplinary nature of Systems Engineering [8]. Interface management is considered as one of the most powerful tools of systems management [9]. The interfaces in mechatronic systems can be used to describe the interactions of subsystems designed by different disciplines. Therefore it is significant to develop a proper interface classification in order to represent much more details of an interface and help the engineers to avoid the confusion by the misuse of interfaces.

Stewarddescribes the interactions of subsystem as "information flows", but such information flows are not described in detail [10]. Counsell et al describe the connections between different components as material, information and power[11]. Sellgrenproposes that interfaces can be classified as attachment, constraint and contact, but his proposition mainly focuses on the mechanical interface [12]. Chen et al classify the interfaces as the "constraints" between electrical/electronic discipline and mechanical discipline, but the interfaces between software discipline and electrical/electronic discipline or mechanical discipline are not mentioned [13]. Pahl et alpropose a method named Modular Product Development (MPD) for complex system. This method starts by decomposing the product into modules. The exchanges of energy, materials, and signal between the modules were mentioned in this method [14]. Liang et al develop a more detailed classification based on the proposition of 
Pahl et al by refining the energy as electrical, mechanical and hydraulic, etc [6]. However, the interface between software and other disciplines are not taken into consideration either. Komotoet al believe that some physical implementations have nothing to do with transformation of energy, material, and signal (e.g., a function to fix connection between two mechanical components or a function that holds a position), but they can be used to connect two components as the interfaces. Thus geometry plays a crucial role during the design process. They point out that attention should be also paid to such geometric information [15]. Sosa et al distinguish the interfaces in terms of spatial dependency, structural dependency, energy dependency, material dependency and information dependency [16]. Such classification method may lead to the misuse of overlapping interfaces. For instance, the material dependency is described as "a requirement related to transferring airflow, oil, fuel, or water". However, such process of material transfer often occurs with the energy transfer which was defined as "energy dependency". Bettiget al point out the interfaces representation problem and tried to identify an overall representational schema. Seven classes of interfaces are firstly suggested: Attachment interface, transfer interface, control and communication interface, power (electrical) interface, spatial interface, field interface and environmental interface. The seven interface classes are reduced to four general classes of interface: attachment interfaces, control and power interfaces, transfer interfaces and field interfaces. The reduced classification defines the field interface as "an interface that transmits energy, material or signal as an unintended side-effect of the intended function of a module". This classification begins to consider the negative effects of interfaces [17]. However, the field interface is so generic and need to be detailed.

As presented before, the interface classification can give much more details about an interface. Such details should be included in the interface data model. The interface data model has been partially represented by current product models. The product model can be used as an effective and efficient technology for the design of mechatronic systems because it includes all the information that can be accessed, stored, served and reused by the stakeholders. The authors reviewed current product models, including STEP, CPM, MOKA and PPO. The interface data model should be created as a part of product model of mechatronic systems. It should not only take into account the proposed interface classification, but also represent the relationship between the interface and other parts of the product model. However, according to the evaluation results of the current product models, the interface data model has not been fully developed in current product models [5].

The interface compatibility can help the designers to ensure consistency among different design teams and to prevent design errors during collaborative design process [18]. However, due to the partially-developed interface data model in current product models, how can the interface data model be used to support the interface compatibility has not been involved.

By considering the drawbacks of current interface classifications and interface data models, the authors will present the proposed multidisciplinary interface modelling approach in next section. 


\section{Multidisciplinary interface modelling approach}

As discussed in previous section, the current interface classifications and interface data models show several drawbacks. Three aspects of the multidisciplinary interface modelling approach, interface classification, interface data model and interface compatibility, will be presented hereafter.

\subsection{Interface classification}

The proposed interface classification for mechatronic systems concerns an interface through three features: Type, Configuration and Desired/undesired.

Type: which types of information are transferred in the interface?Four types of information are proposed: geometric interface, energy interface, control interface and data interface.

Configuration: which elements does the interface link?Five configurations of interfaces are proposed: interface between components (C-I-C), interface between a component and the environment (C-I-E), interface between a component and an interface (C-I-I), interface between two interfaces (I-I-I) and interface between an interface and the environment (I-I-E).

Desired/undesired: does the interface create positive effects or negative effects to the whole system?

The interface classification is considered as the foundation of interface modelling, because this classification not only gives much more details of an interface, but also avoids the confusion by the misuse of interfaces [17]. In next sub-section, a new interface data model based on the classification will be presented.

\subsection{Interface data model}

The second aspect of the multidisciplinary interface is the interface data model. The interface classification previously presented should be represented by the interface data model. Moreover, attention should be paid to the concept of "port". In the context of multidisciplinary interface modelling of mechatronic systems, the port refers to the primary location through which two elements interact with each other [6]. Two attributes of port will be introduced. The first attribute is called "direction", which represents how the information is transferred through this port. In other words, the direction of a port holds a definition on which one is the master and which one is the slave of the two elements linked by the interface. A compartment listing the attributes (in, out and in/out) is to indicate that the information flows in (out of or in $\&$ out of) the element through the port. The second attribute of the class port is the visibility. This attribute is used to describe how the port can be accessed. The authorized values are "public", "protected" and "private". The parameter and document related with one public port is accessible directly by any engineers from any disciplines during the design process. A protected port can only be accessed by the creators and the authorised engineers from other disciplines. The port carrying the private property is accessible only by those who design it. 
Fig. 1 shows interface data model represented as a UML class diagram ${ }^{1}$. On the one hand, the interface classification and the port with its related attributes are represented by the Interface and the Port class. On the other hand, it represents the relationship between the interface and other elements. However, main entities of current product models can be found in the proposed interface data model so that mapping can be constructed between current product models and the interface data model. Therefore the interface data model can be used as an extension of current product models.

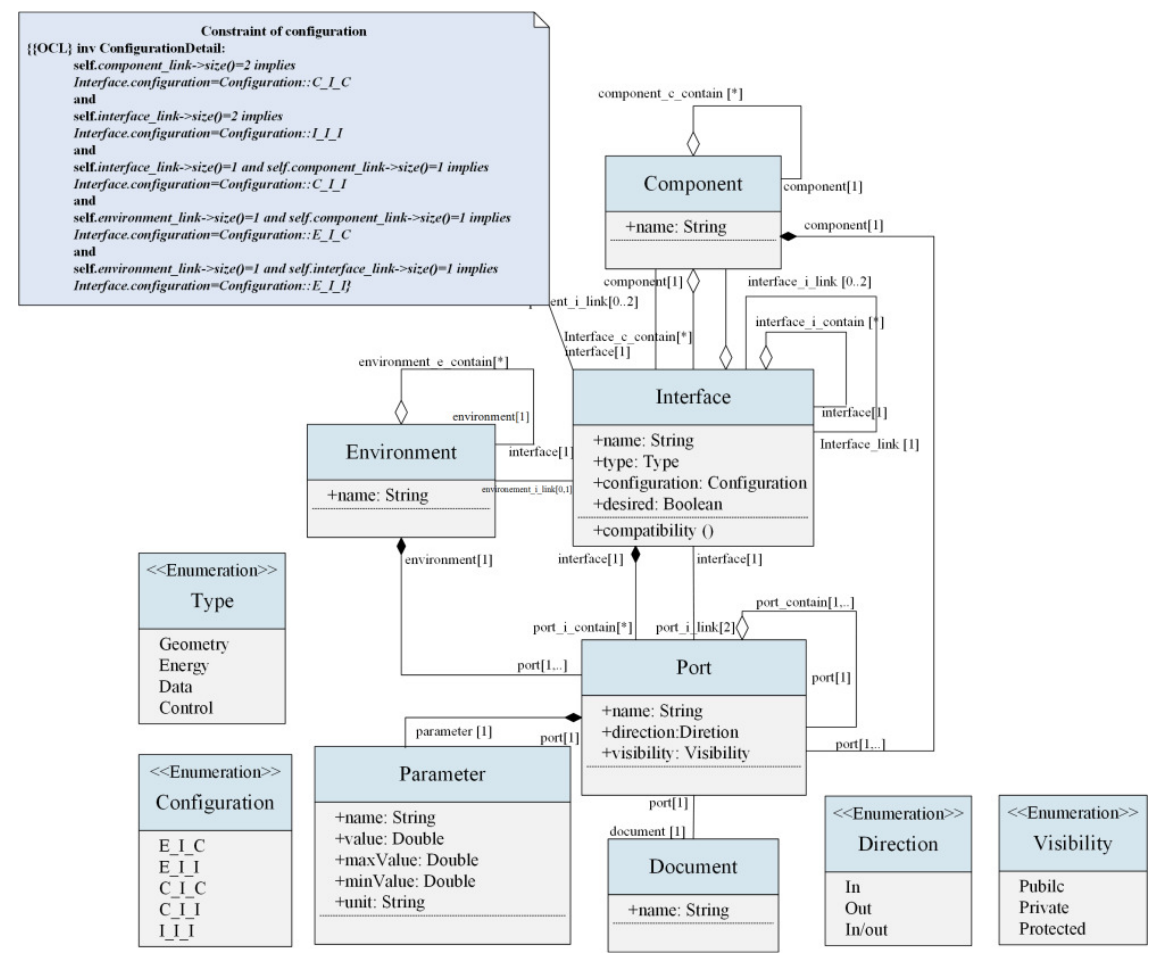

Fig. 1. UML class diagram of interface

\subsection{Interface compatibility}

The last aspect of the interface modelling approach is the interface compatibility. The authors propose two rules to test the interface compatibility. Once the data model of an interface is instantiated, the interface compatibility should be checked by the rules. One example is cited here to illustrate the compatibility test method. Two components (Component 1 and Component 2) are connected by an interface (Interface) through the ports ( $\mathrm{CP} 1$ and $\mathrm{CP} 2)$. Once the data model of the interface has been instantiated, the interface compatibility should be checked. Two compatibility rules are presented as follows:

${ }^{1}$ http://www.uml.org/ 
CP1.Parameters1.value $=$ CP2.Parameters 2 . value

CP1. Parameters1. unit $=C P 2$. Parameters 2 . unit $(1)$

\author{
CP1. Parameters1.value $<$ CP2. Parameters 2 . maxValue \\ CP1. Parameters1. value $>$ CP2. Parameters 2 . minValue \\ CP1.Parameters1. unit $=C P 2$. Parameters 2 . unit
}

In the compatibility Rule (1), CP1.Parameterl presents the parameter stored in the class Parameter of the port $\mathrm{CP} 1$, and CP2.Parameter 2 is the parameter of port CP2. In order to ensure the two components integrate with each other correctly, both the value and the unit of the parameters of CP1 and CP2 should be equal. The Rule (2) is applied to two cases. The first case is that sometimes the design parameter of one port is not expressed by an exact value accurately but described as a constraint, such as the minimum input current, maximum diameter of the hole and etc. The second case concerns the component tolerance. Component can hardly hold dimensions precisely to the nominal value, so there must be an acceptable degree of variation.

Once an interface has been established, the compatibility should be checked by the compatibility rules presented above. If one interface proves to be incompatible, two incompatibility solutions can be adopted to deal with this incompatible interface. These solutions will be presented as follows:

Fig. 2 shows an example of the two incompatibility solutions. A simple mechatronic system (System) can be decomposed into 2 components (Component 1 and Component 2) and an interface (Interface 1). However, when the designs of the two components have been finished by the designers, the interface (Interface1) may prove to be incompatible. Fig. 2(a) shows the first solution. The Component 2 can be redesigned and replaced by the Component 3 . The compatibility of the interface (Interface 1) should be then re-checked. Fig. 2(b) shows the second solution. A new component (Component 3) can be added between the two components and two new interfaces (Interface 1.1 and Interface 1.2) will be created accordingly. The compatibilities of the two new interfaces should be checked.

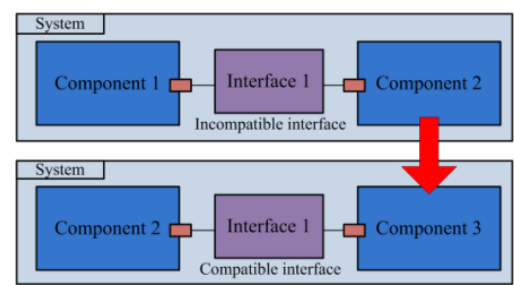

(a) Solution 1

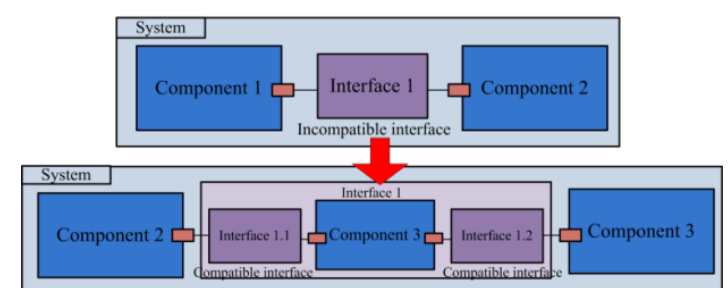

(b) Solution 2 
Fig. 2. Example of incompatibility solutions

Solution 1 is simple to operate by the designers because this solution demands the designers to change one of the two component linked by the incompatible interface. However, in a complex mechatronic system, one component may link to others through several interfaces. After the change of such component to solve the incompatibility problems of one interface, nevertheless, other interfaces linked to this component which proved to be compatible before may become incompatible. Such conflict may always exist during the design process. Solution 2 demands the designers to further decompose the incompatible interface, which is much more complex than Solution 1. However, Solution 2 does not create design conflicts because the new component does not affect other elements of the system. Therefore, the designers should carefully select the solutions to solve the incompatibility problems during the design process.

The three aspects of the multidisciplinary interface modelling approach, have been presented previously in order to help the engineers achieve the multidisciplinary integration during the design process of mechatronic systems. The authors also provide a case study by means of a three dimensional (3D) measurement system to demonstrate the propositions more clearly in next section.

\section{Case study}

The case study is chosen to demonstrate the proposed interface modelling approach based on a three dimensional (3D) measurement system [19]. This measurement system is designed for reconstruction of object surface based on optical measurement. It is considered as a mechatronic system integrating synergistically the electrical/ electronic system, mechanical parts and information processing and optical technology. Fig. 3 shows the principle of the measurement modes.

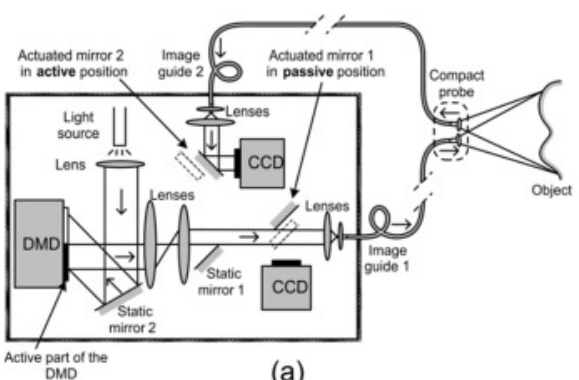

(a)

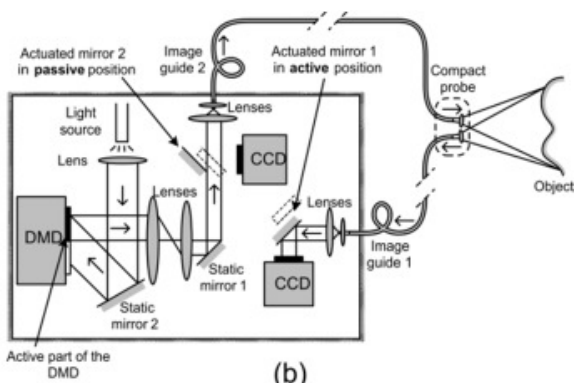

(b)

Fig. 3. Principle of the measurement modes: (a) Active mode 1 (b) Active mode 2

This 3D measurement system can be generally decomposed into six sub-systems. Pattern projection sub-system $(\mathrm{C} 1.1)$ projects the fringe patterns onto the measured object, and the deformed image reflected by the measured object is received by the Deformed image reception sub-system (C1.2). The original fringe patterns and the deformed image will be compared and analysed by the $3 \mathrm{D}$ image reconstruction sub- 
system (C1.3). The measurement modes (Fig.3) can be switched by the Mode switch sub-system (C1.4). The whole system is supported by the Mechanical support sub-system (C1.5) while the power is supplied by the Power supply sub-system (C1.6). In order to demonstrate the proposed data modelling approach more clearly, the Pattern projection subsystem $(\mathrm{C} 1.1)$ has been selected in this paper.

The Pattern projection sub-system is one of the most important parts in the 3D measurement system. A white light source (C1.11) illuminates the DMD (Digital Micro-mirror Device) (C1.12) generating the fringe patterns. The fringe patterns are then injected into the image guide $(\mathrm{C} 1.13)$ and projected on the measured object through the compact probe (C1.14).

This image guide $(\mathrm{C} 1.13)$ has to be taken into consideration very carefully. On the one hand, an image guide with a high resolution is needed to meet the requirement of the inspection for the industrial equipments. On the other hand, the image guide has to be flexible enough to accommodate the industrial environment (E1). Therefore an interface (I1.1) can be constructed between the industrial environment (E1) and the image guide $(\mathrm{C} 1.13)$. In order to realise a better reconstruction result, the designers of the optical team choose the image guide with the highest resolution (FIGH-100$1500 \mathrm{~N}$ ), whose minimum bending radius is $200 \mathrm{~mm}$. By analysing the scale of the whole measurement system and the industrial environment, the designers of the mechanical team propose the maximum scale of system. The value of the maximum scale $(350 \mathrm{~mm})$ is represented as maxValue in the instance Industrial scale.

The data model of the interface I1.1 with the ports EP1 and CP2 can be created according to the above analysis. The UML object diagram in Fig.4 shows the instance of the interface I1.1 and the two ports EP1 and CP2 linked by I1.1. Demonstrator based on the data model has been developed by making use of CATIA V6. The interface compatibility rules proposed in Section 3.3 is realised by the Knowledgeware of CATIA V6. The check result of interface compatibility shows that the interface between the industrial environment and the image guide is incompatible, because the minimum bending diameter of the image guide is beyond the size limit proposed by the industrial environment.

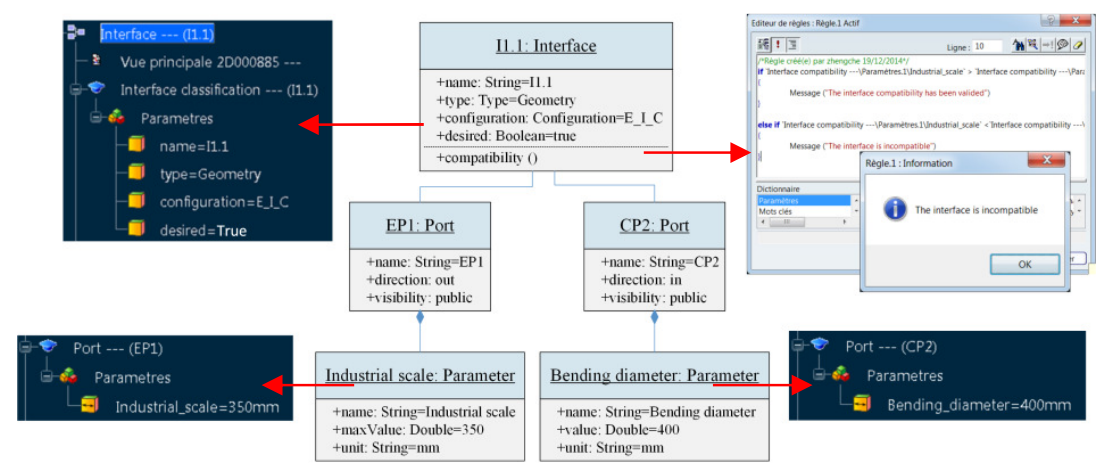

Fig.4. Instance of the incompatible interface I1.1

The incompatibility of the interface between the industrial environment and the image guide has been detected. The incompatibility solutions proposed in Section 3.3 can help the designers to solve this problem. As described in the Solution 1, one 
element linked by the incompatible interface can be changed to solve this incompatibility problem. Thus, by referring the data sheet of the image guide, the designers of the optical team replace the image guide with FIGH-70-1300N whose minimum bending radius is $150 \mathrm{~mm}$, and the interface compatibility can then be validated. This compatible interface between the industrial environment and the image guide means that the size of the image guide accommodates the industrial environment.

Once the design of the image guide has been finished (FIGH-70-1300N), the attention should be paid to the interface (I1.12) between the DMD (C1.12) and the image guide $(\mathrm{C} 1.13)$. By referring the data sheet of the image guide, the designers of the optical team can find that the image circle diameter of the FIGH-70-1300N is $1.2 \mathrm{~mm}$. However, the maximum diameter of the image circle projected by the DMD should be the width of the DMD $(8.3 \mathrm{~mm})$, which can be obtained by calculating from the data sheet of the DMD. By applying the interface compatibility test method, the designers will find that the interface between the DMD and the image guide is incompatible because the image circle diameter of the DMD is different from that of the image guide. This incompatible interface between the DMD and the image guide indicates that these two components do not integrate correctly and cannot be connected with each other directly. Solution 2 requires the incompatible interface to be decomposed into an Interface-Component-Interface structure. A lenses system which can change the image circle diameter will be designed by the designers of the optical team. The compatibility of the new interface will be then checked.

The sub-system called Pattern projection sub-system of the 3D measurement system has been selected to demonstrate the proposed multidisciplinary interface modelling approach. Once the data model of an interface has been initiated, the interface compatibility should be checked in order to guarantee the different elements integrate correctly.

\section{Conclusion}

The paper focuses on the multidisciplinary interface modelling approach which can be used during the collaborative design process of mechatronic systems to help the designers achieve the multidisciplinary integration. This approach incorporates the interface classification, the interfaces data model and the interface compatibility. The interface classification provides much more details of an interface to the designers and helps them to avoid the confusion by the misuse of interfaces. The interface data model will be created as a part of product model of mechatronic systems. It not only takes into account the information proposed by the interface classification, but also represents the relationship between the interface and other parts of the product model. In order to guarantee the different elements integrate correctly and eventually ensure the multidisciplinary integration among design teams, interface compatibility should be checked with the support of interface data model.

Acknowledgement. This work has been partially supported by the Doctoral Program of Chinese Scholarship Counciland the Austrian Center of Competence in 
Mechatronics (ACCM) / Linz Center of Mechatronics (LCM) in the framework of the Austrian COMET program. It also takes place in the scientific strategy of Labex MS2T supported by the ANR - French National Agency for Research.

\section{References}

1. Carryer, J., Ohline, R., Kenny, T.: Introduction to Mechatronic Design. Prentice Hall, Boston (2011)

2. Sohlenius, G.: Concurrent Engineering. CIRP Ann - Manuf Technol. 4, 645--655 (1992)

3. Lefèvre, J., Charles, S., Bosch-Mauchand, M., Eynard, B., Padiolleau, E.: Multidisciplinary Modelling and Simulation for Mechatronic Design. J Des Res. 12, 127-144 (2014)

4. Abramovici, M.,Bellalouna, F.: Service Oriented Architecture for the Integration of Domain-Specific PLM Systems within the Mechatronic Product Development. In: 7th International Symposium on Tools and Methods of Competitive Engineering, pp. 941--953. Izmir (2008)

5. Zheng, C., Bricogne, M., Le Duigou, J., Eynard, B.: Survey on Mechatronic engineering: A Focus on Design Methods and Product Models. Adv Eng Informatics. 28, 241--257 (2014)

6. Liang, V.C., Paredis, C.J.J A Port Ontology for Conceptual Design of Systems. J ComputInfSci Eng. 4, 206-217 (2004)

7. Dorfman, M.,: Systems and Software Requirements Engineering. In: IEEE Computer Society Press Tutorial, pp.7--22. IEEE Computer Society Press, Los Alamitos (1990)

8. Fosse, E., Delp, C.L.: Systems Engineering Interfaces : A Model Based Approach. In: IEEE Aerospace Conference, pp. 1--8. Big Sky (2013)

9. Blyler, J.: Interface management. InstrumMeas Mag. 7, 32--37 (2004)

10.Steward, D. V. Partitioning and Tearing Systems of Equations. J SocIndAppl Math. 2, 345-$365(1965)$

11.Counsell, J., Ian, P., David, D., Duffy, M.: Schemebuilder: Computer Aided Knowledge Based Design of Mechatronic Systems. AssemAutom. 19, 129--138 (1999)

12.Sellgren, U.: Interface Modeling - A Modular Approach to Identify and Assess Unintended Product Behavior. In: NAFEMS 2nd Nordic Seminar: Prediction and Modelling of Failure Using FEA. Roskilde (2006)

13.Chen, K., Bankston, J., Panchal, J.H., Schaefer, D.: A Framework for the Integrated Design of Mechatronic Systems. In: Collaborative Design and Planning for Digital Manufacturing, pp 37--70. Springer, London (2009)

14.Pahl, G., Beitz, W., Feldhusen, J., Grote, K.H.: Engineering Design: A Systematic Approach. Springer-Verlag, London (2007)

15.Komoto, H., Tomiyama, T.: A Framework for Computer-Aided Conceptual Design and Its Application to System Architecting of Mechatronics Products. Comput Des. 44, 931--946 (2012)

16.Sosa, M.E., Eppinger, S.D., Rowles,C.M.: Designing modular and integrative systems. In: ASME 2000 International Design Engineering Technical Conferences and Computers and Information in Engineering Conference. Baltimore (2000)

17.Bettig, B., Gershenson, J.K.: The Representation of Module Interfaces. Int J Prod Dev. 10, 291--317, 2010

18.Rahmani, K., Thomson, V.: Ontology based Interface Design and Control Methodology for Collaborative Product Development. Comput Des. 44, 432--444 (2012)

19.Hou, Y., Dupont, E., Petit, L., Redarce, T., Lamarque, F.:Dynamic reconfiguration of a compact active stereovision system with digital electromagnetic actuators. In: IEEE/ASME International Conference on Advanced Intelligent Mechatronics (2014) 